\title{
OPS/OBS Scheduling Algorithms: Incorporating a Wavelength Conversion Cost in the Performance Analysis
}

Kurt Van Hautegem, Wouter Rogiest \& Herwig Bruneel

\{kurt.vanhautegem $\bowtie$, wouter.rogiest, herwig.bruneel\}@telin.UGent.be

Ghent University, Belgium (UGent)

Department of Telecommunications and Information Processing (TELIN) Stochastic Modelling and Analysis of Communication Systems (SMACS) 


\section{Overview presentation}

- The optical backbone

- Contention resolution \& scheduling basics

- Scheduling algorithms

- Performance results

- Energy consumption results

- Conclusions 


\section{Overview presentation}

- The optical backbone

- Contention resolution \& scheduling basics

- Scheduling algorithms

- Performance results

- Energy consumption results

- Conclusions 


\section{The optical backbone}

technological developments \& internet-based business models

$\Rightarrow$ demand for bandwidth $>$

\section{connections}

links

optical fibers

'unlimited' capacity

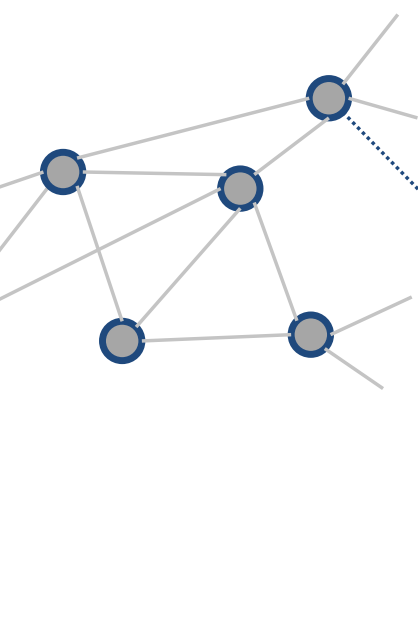

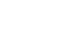

intersections

nodes

switches

$3 / 27$

bottleneck 


\section{Introduction: the optical backbone}

Currently: circuit switching

- dedicated communication channel

$\checkmark$ guaranteed packet arrival

$\checkmark$ fixed delay

$x$ inefficient use of available fiber capacity ( bandwidth)
Future: packet-based switching

- shared links

$\checkmark$ improved usage of capacity

- contention possible

$\rightarrow \times$ potentially, packet loss

$x$ no fixed delay

x potentially, substantial delay

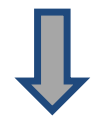

main motivation of this

contention resolution 


\section{Overview presentation}

- The optical backbone

- Contention resolution \& scheduling basics

- Scheduling algorithms

- Performance results

- Energy consumption results

- Conclusions 


\section{Overview presentation}

- The optical backbone

- Contention resolution $\&$ scheduling basics

- Scheduling algorithms

- Performance results

- Energy consumption results

- Conclusions 


\section{Contention \& resolution}

contention:

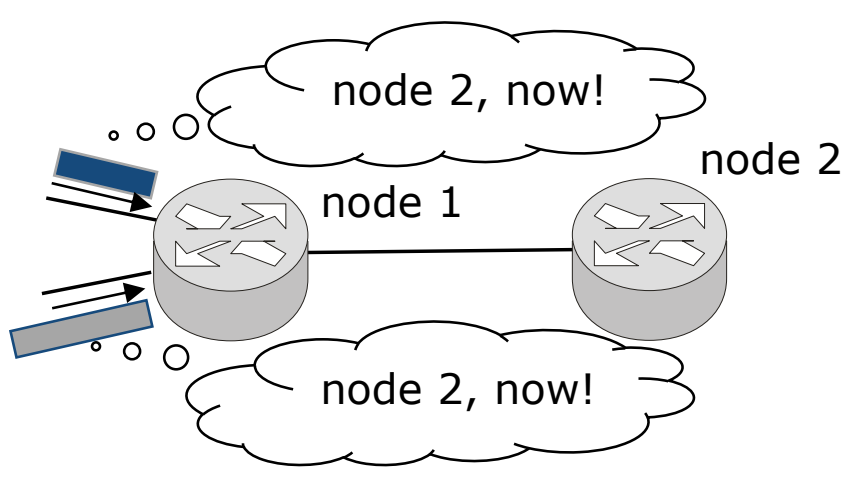

straightforward solution: electronic buffering (RAM)

x cannot keep up with optical speeds

$x$ energy consuming $\mathrm{O} / \mathrm{E} / \mathrm{O}$ conversions 


\section{Optical contention resolution: two means}

(1)

wavelength converters (WCs)

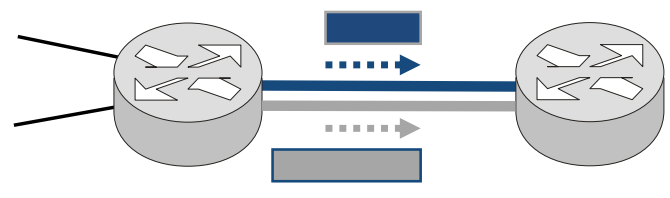

- c wavelengths

- unlimited wavelength conversion capacity

- energy consuming
(2)

Fiber Delay Lines (FDLs)

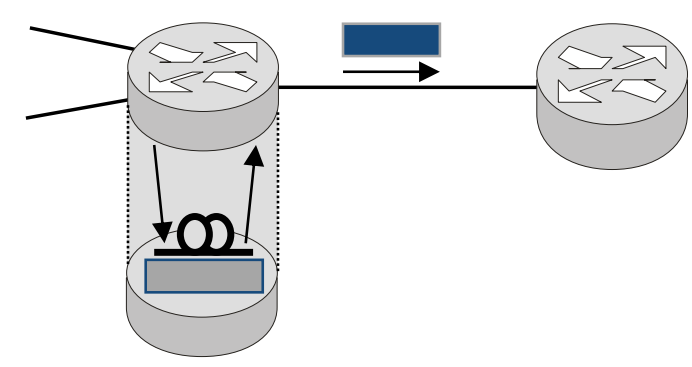

- set of fibers, $\#=\mathrm{N}+1 \cdot \mathrm{N}=$ buffer size

- lengths $j \cdot D, j=0 \ldots N \quad \bullet \quad D=$ granularity 


\section{Provisional schedule}

wavelength conversion + Fiber Delay Lines $\Rightarrow$ provisional schedule

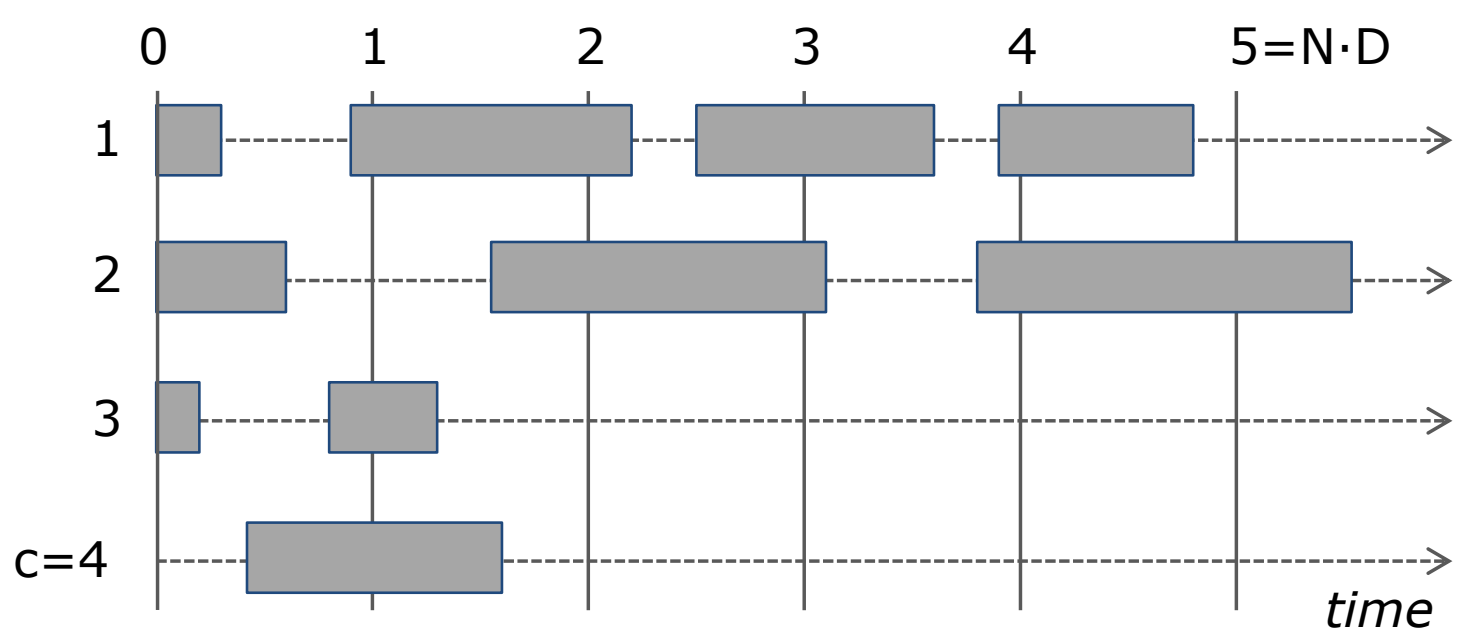

- shows already scheduled packets

- updated at every arrival

- horizontal lines (dotted): outgoing wavelengths $(c=4)$

- vertical lines: delays of FDLs $(N=5, D=1)$ 


\section{Schedule for minimal loss}

\section{SCHEDULING}

\section{choose:}

- outgoing wavelength $\mathrm{i}(\mathrm{i}=1$...c)

- delay line $j(j=0 . . . N)$

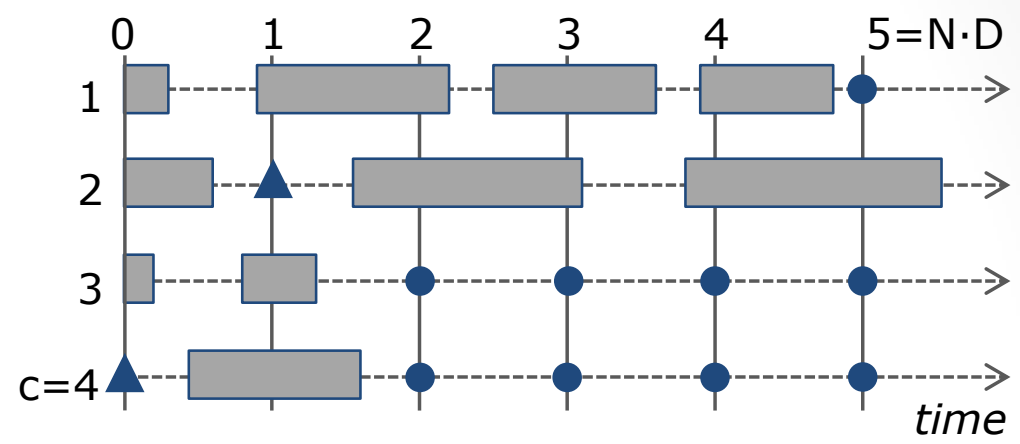

constraints:

- no overlap

- type of algorithm

- non-void-filling (NVF) •

minimize loss probability (LP)

- void-filling (VF) $\bullet+\Delta$

\section{goal:}

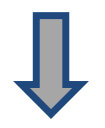

choose SP "wisely"

satisfied: Scheduling Points (SPs) 


\section{Overview presentation}

- The optical backbone

- Contention resolution \& scheduling basics

- Scheduling algorithms

- Performance results

- Energy consumption results

- Conclusions 


\section{Overview presentation}

- The optical backbone

- Contention resolution \& scheduling basics

- Scheduling algorithms

- Performance results

- Energy consumption results

- Conclusions 


\section{Scheduling algorithms: current}

- JSQ(-NVF)

join the shortest queue

=wavelength with shortest horizon

- D-G(-NVF) (b)

first priority: minimum delay

second priority: minimum gap

- $\quad$ G-D(-NVF)

first priority: minimum gap

second priority: minimum delay

- D-G-VF (d) packet lenght $\leq 0,4$

(e) $0,4<$ packet length $\leq 0,6$

(b) packet length $>0,6$

- G-D-VF (d) packet length $\leq 0,4$

(c) packet length $>0,4$
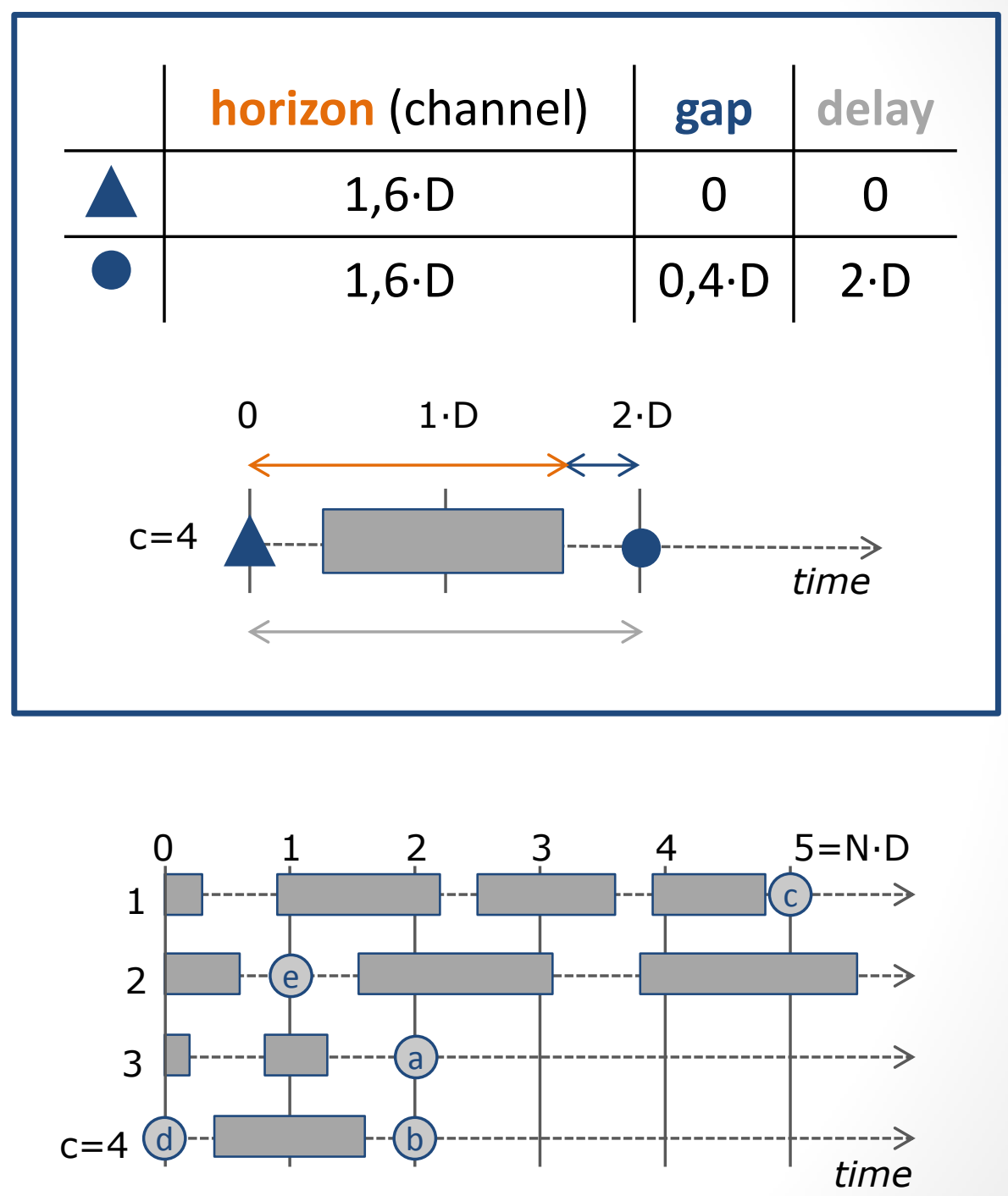


\section{Scheduling algorithms: new}

- assign cost to each SP

- choose SP with lowest cost

\section{2 cost functions}

C: cost of SP taking into account gap and delay:

$$
C=\alpha \cdot g a p+(1-\alpha) \cdot d e l a y
$$

CW: cost of SP taking into account gap, delay and wavelength conversion:

$$
C W=\left(\frac{1}{1+\beta}\right)^{1-\delta_{w i}} \cdot[\alpha \cdot g a p+(1-\alpha) \cdot \text { delay }]+\frac{\beta}{1+\beta} \cdot D \cdot\left[1-\delta_{w i}\right]
$$




\section{Scheduling algorithms: $C$}

C: cost of SP (gap and delay, not wavelenth conversion):

$$
C=\alpha \cdot \operatorname{gap}+(1-\alpha) \cdot \text { delay }
$$

- $\alpha$ : algorithm parameter to optimise for minimal loss probability

- weighted average of gap and delay

- MOTIVATION: propose algorithms with better performance

$\Rightarrow$ algorithms C(-NVF) and C-VF 


\section{Scheduling algorithms: $C W$}

CW: cost of SP (gap, delay and wavelength conversion):

$$
C W=\left(\frac{1}{1+\beta}\right)^{1-\delta_{w i}} \cdot[\alpha \cdot g a p+(1-\alpha) \cdot \text { delay }]+\frac{\beta}{1+\beta} \cdot D \cdot\left[1-\delta_{w i}\right]
$$

- $\alpha$ : algorithm parameter to optimise for minimal LP

- $\quad \beta$ : algorithm parameter to reduce wavelength conversion ( energy consumption)

- i: outgoing wavelength, w: incoming wavelength, $\delta_{\mathrm{wi}}$ : Kronecker's delta

- $\neq C$, due to extra summand to penalise use of wavelength converter

- MOTIVATION: propose algorithms with reduced energy consumption 


\section{Overview presentation}

- The optical backbone

- Contention resolution \& scheduling basics

- Scheduling algorithms

- Performance results

- Energy consumption results

- Conclusions 


\section{Overview presentation}

- The optical backbone

- Contention resolution \& scheduling basics

- Scheduling algorithms

- Performance results

- Energy consumption results

- Conclusions 


\section{Performance results: assumptions}

- $\mathbf{c}=\#$ incoming wavelengths $=\#$ outgoing wavelengths = 4

- $\mathbf{N + 1}=$ \# Fiber Delay Lines $=10$

- inter-arrival time packets = exponentially distributed, $E[T]$

- packet size $=$ exponentially distributed, $E[B]=100$

- arriving wavelength = uniformly distributed

- load $=\rho=\frac{E[B]}{c \cdot E[T]}=80 \%$

- $\mathbf{D}=$ granularity $=10,20, \ldots, 200$

- Monte Carlo simulation 


\section{Performance results: current algorithms}

Loss probability

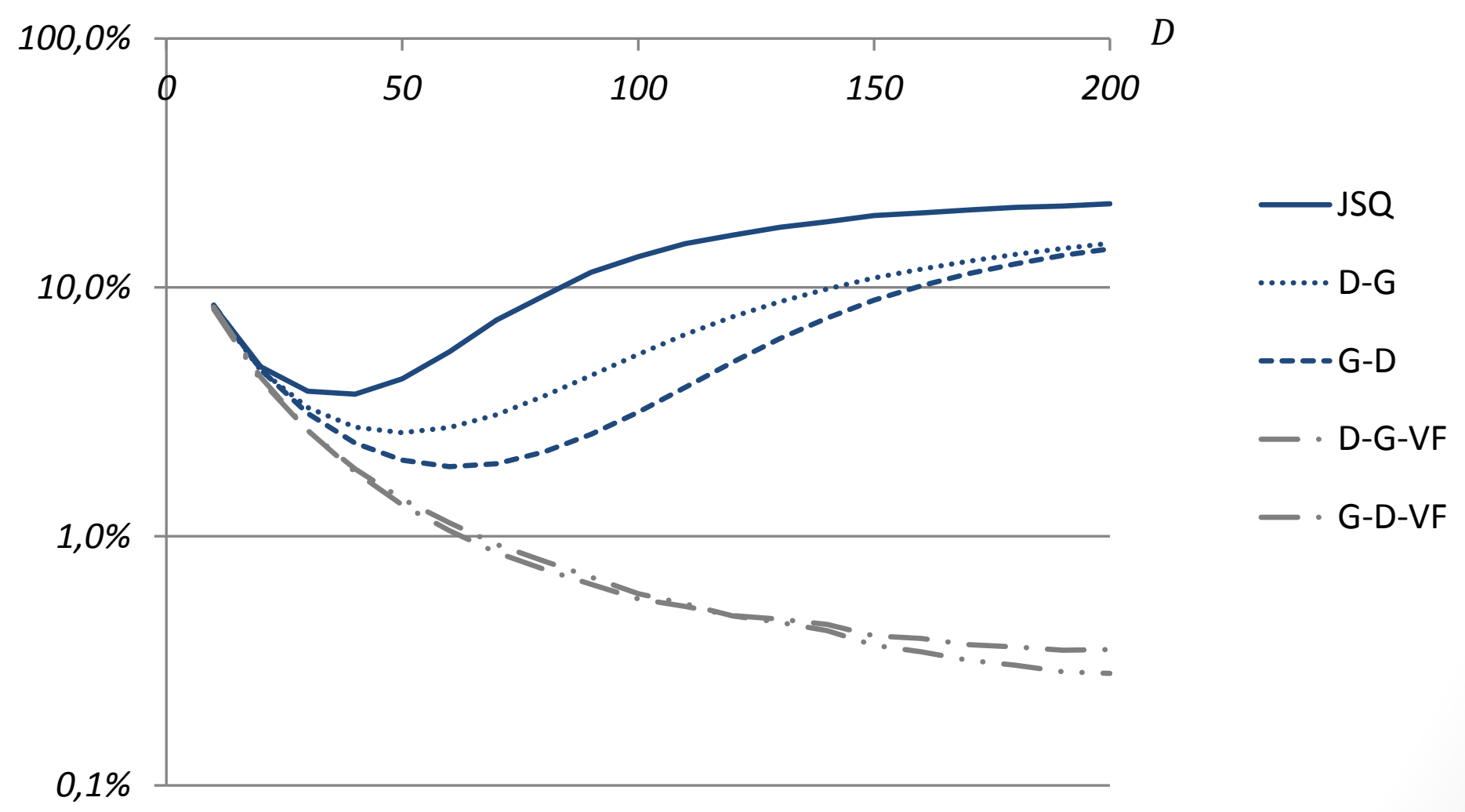




\section{Performance results: C}

$C=\alpha \cdot g a p+(1-\alpha) \cdot d e l a y$

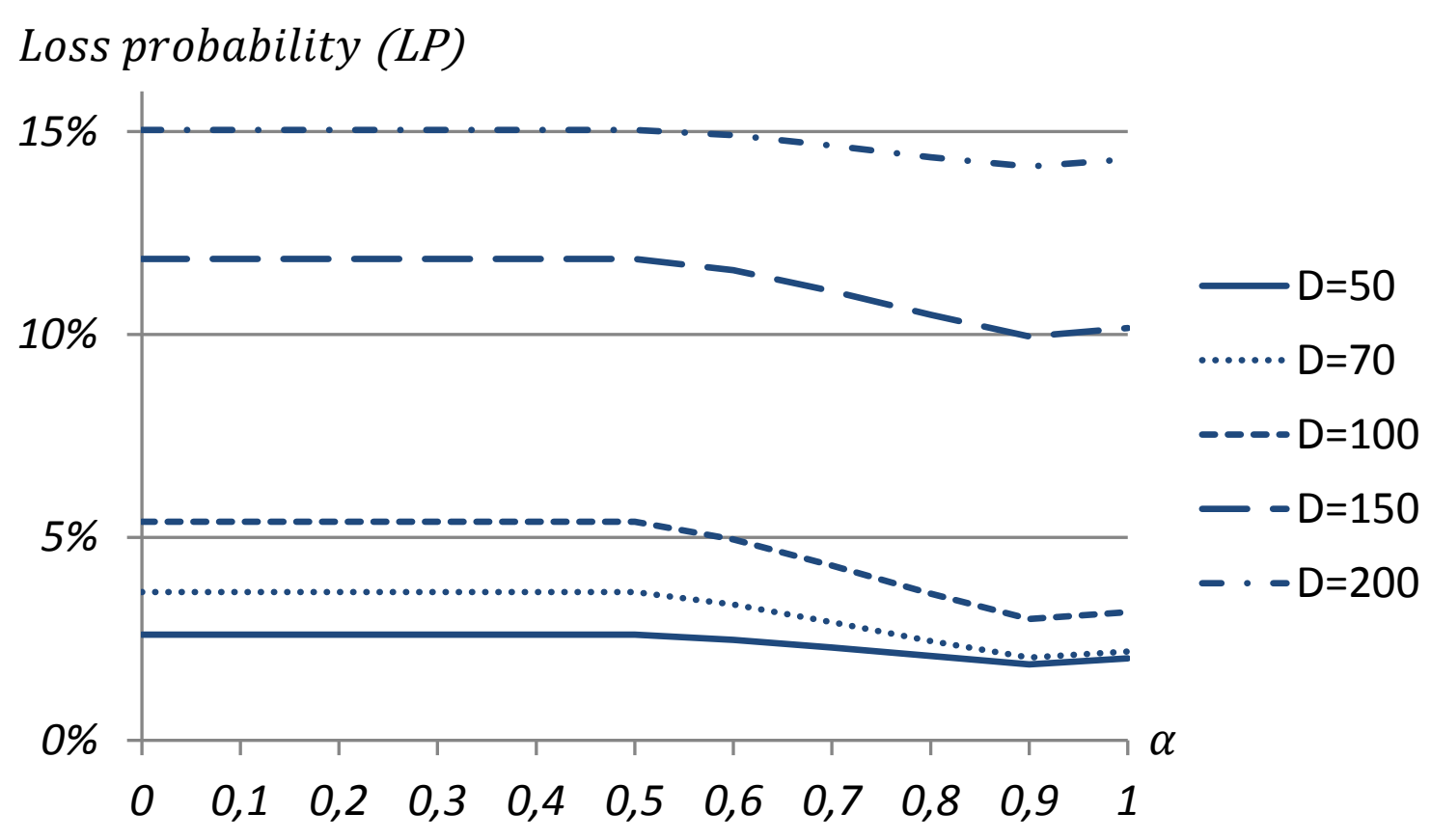

\begin{tabular}{c|ccccc}
$\mathrm{D}$ & 50 & 70 & 100 & 150 & 200 \\
\hline LP reduction (\%) & 7 & 7 & 5 & 2 & 1 \\
$\alpha$ & 0,9 & 0,9 & 0,9 & 0,9 & 0,9
\end{tabular}




\section{Performance results: C-VF}

$C=\alpha \cdot g a p+(1-\alpha) \cdot$ delay

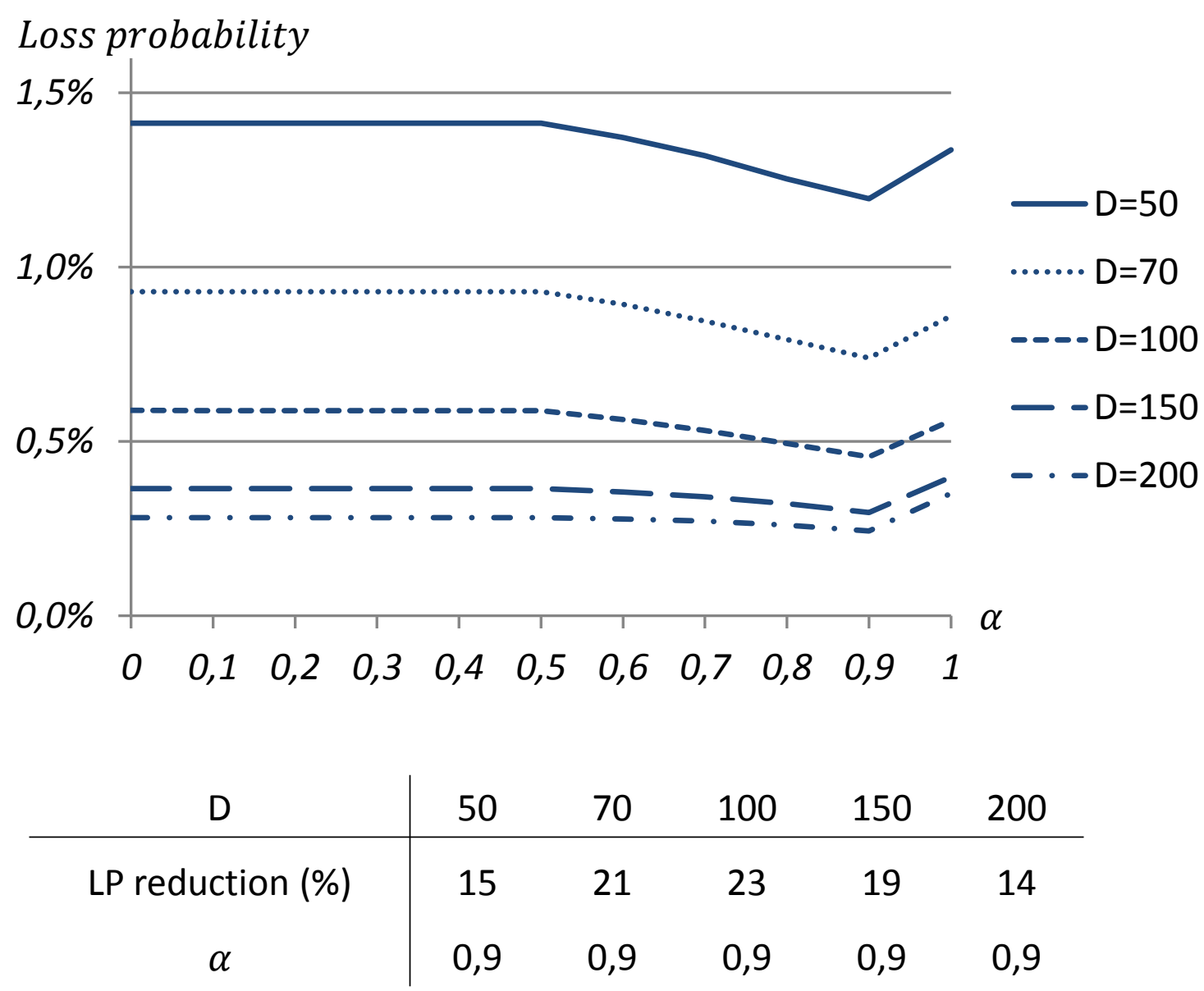




\section{Overview presentation}

- The optical backbone

- Contention resolution \& scheduling basics

- Scheduling algorithms

- Performance results

- Energy consumption results

- Conclusions 


\section{Overview presentation}

- The optical backbone

- Contention resolution \& scheduling basics

- Scheduling algorithms

- Performance results

- Energy consumption results

- Conclusions 


\section{Energy consumption results}

wavelength converters assumed only switched on when converting

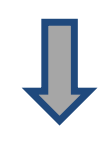

energy consumption $\sim$ payload converted packages

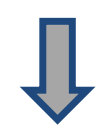

energy consumption measure $=\frac{\text { payload converted packages }}{\text { payload packages not lost }}$ 


\section{Energy consumption results}

Energy consumption measure

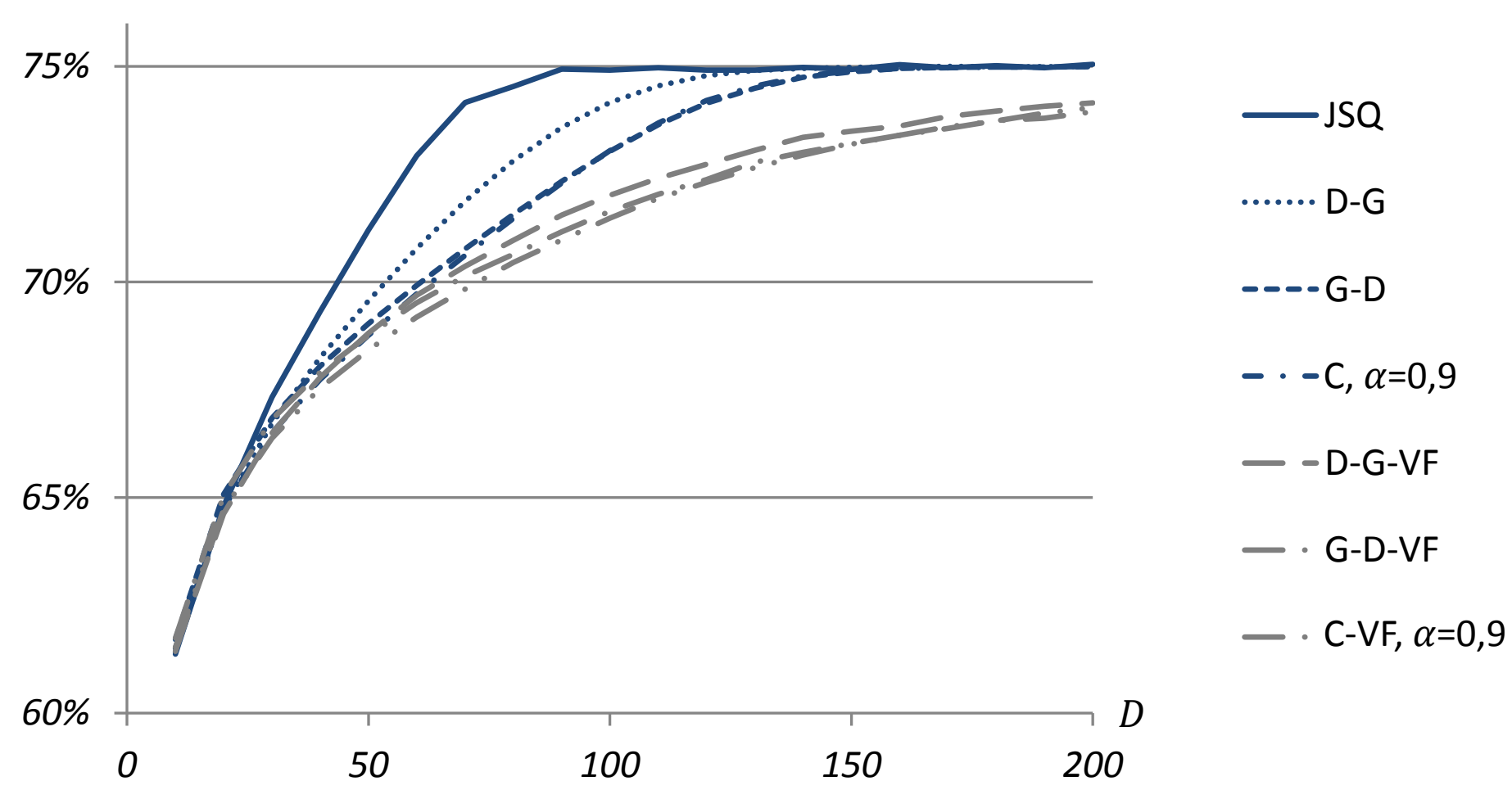




\section{Energy consumption results: $\mathrm{CW}$}

$C W=\left(\frac{1}{1+\beta}\right)^{1-\delta_{w i}} \cdot[\alpha \cdot g a p+(1-\alpha) \cdot$ delay $]+\frac{\beta}{1+\beta} \cdot D \cdot\left[1-\delta_{w i}\right]$

- $\alpha=0,9$

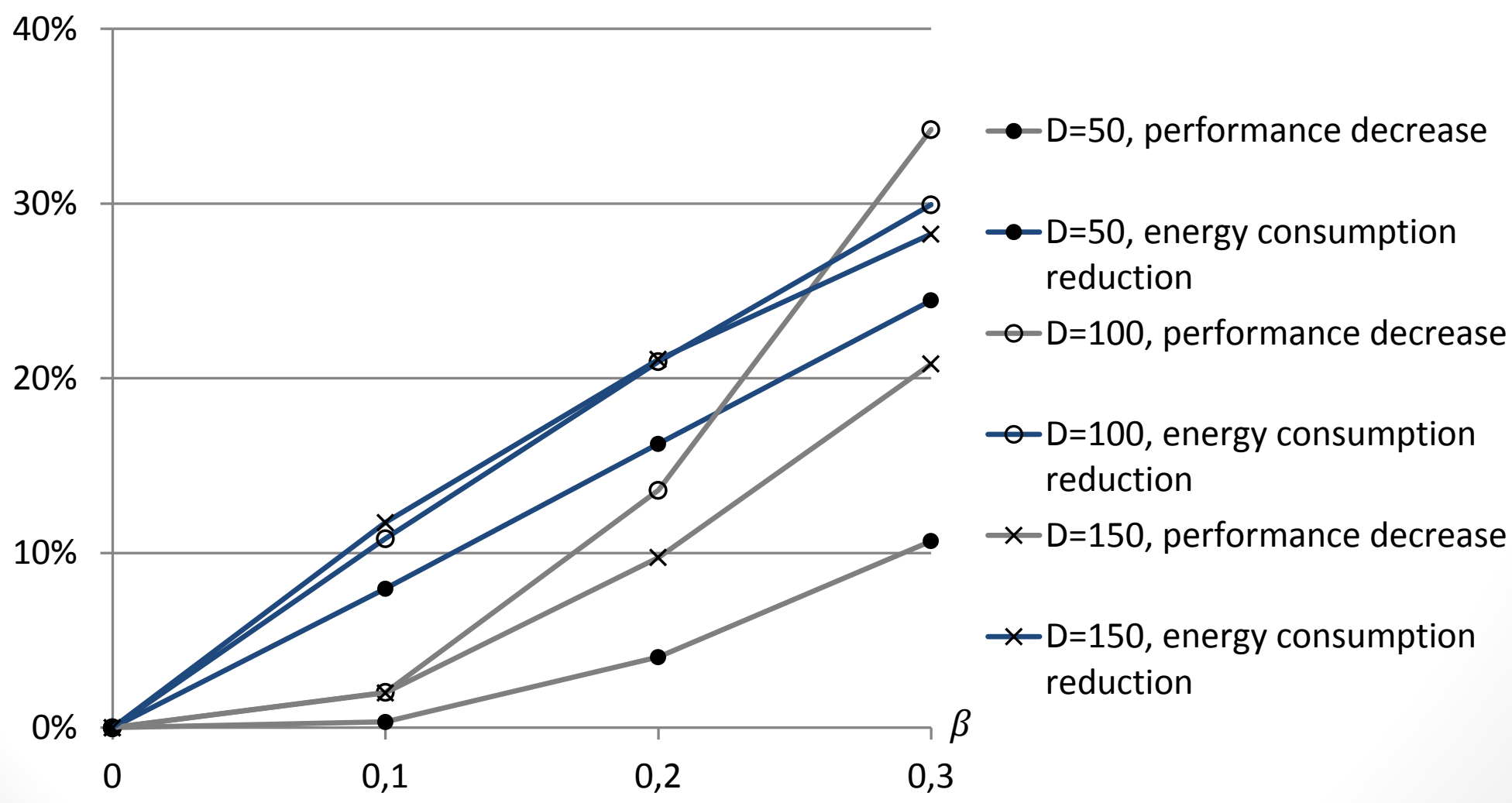




\section{Energy consumption results: $\mathrm{CW}-\mathrm{VF}$}

$C W=\left(\frac{1}{1+\beta}\right)^{1-\delta_{w i}} \cdot[\alpha \cdot g a p+(1-\alpha) \cdot$ delay $]+\frac{\beta}{1+\beta} \cdot D \cdot\left[1-\delta_{w i}\right]$

- $\alpha=0,9$

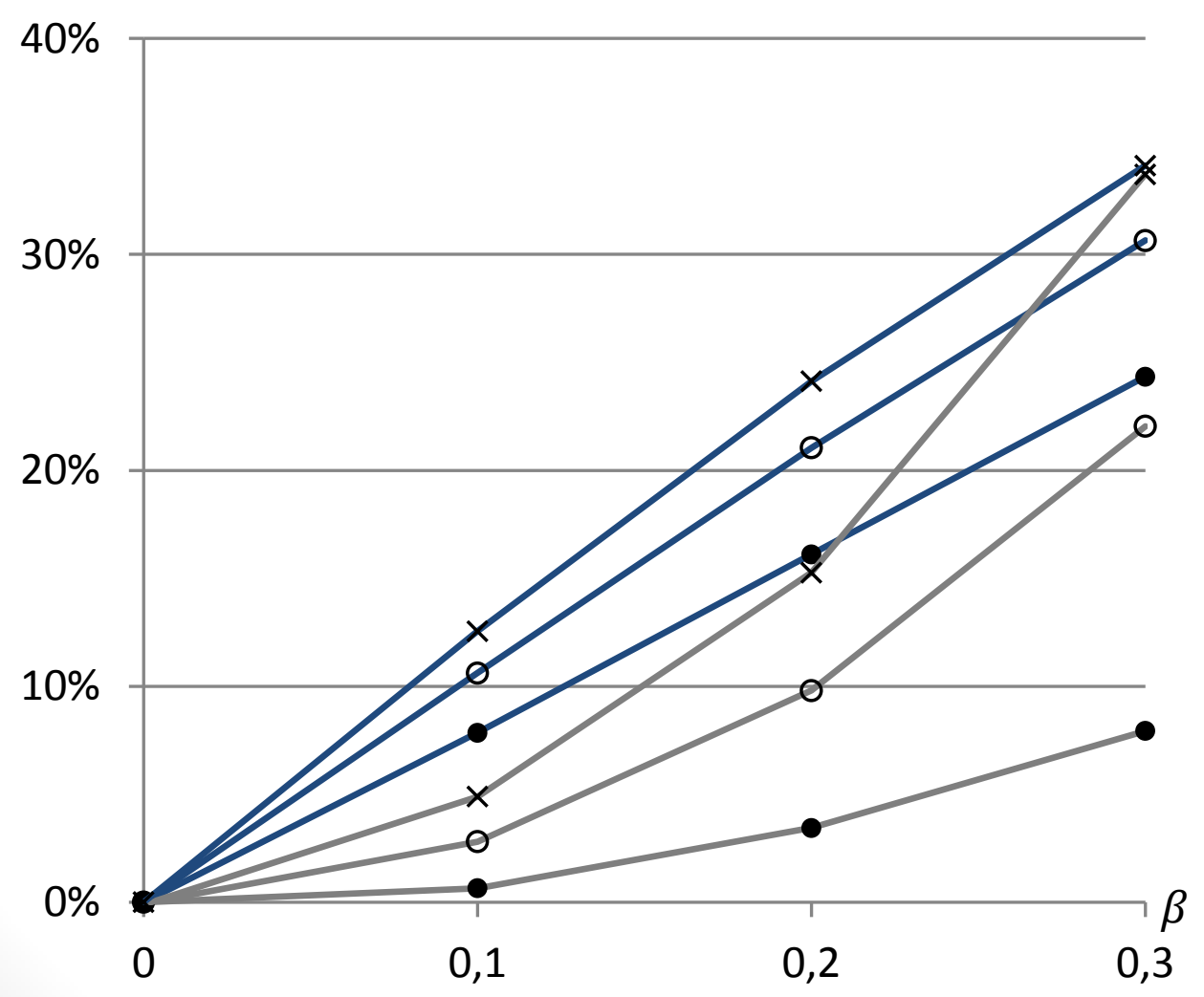

$\bullet D=50$, performance decrease

$\rightarrow D=50$, energy consumption reduction

- $D=100$, performance decrease

$-D=100$, energy consumption reduction

$\star D=150$, performance decrease

$* D=150$, energy consumption reduction 


\section{Overview presentation}

- The optical backbone

- Contention resolution \& scheduling basics

- Scheduling algorithms

- Performance results

- Energy consumption results

- Conclusions 


\section{Overview presentation}

- The optical backbone

- Contention resolution \& scheduling basics

- Scheduling algorithms

- Performance results

- Energy consumption results

- Conclusions 


\section{Conclusions}

cost based approach

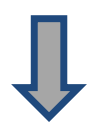

2 cost functions

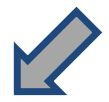

C
- weighted average delay \& gap

- $\alpha$ optimised

- $\quad$ C and C-VF algorithms

- improved performance
CW

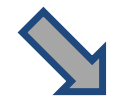

- weighted average delay \& gap + penalty cost for using WC

- fixed optimal $\alpha$, varying $\beta$

- CW and CW-VF algorithms

- improved performance tradeable for energy consumption reduction 
Questions

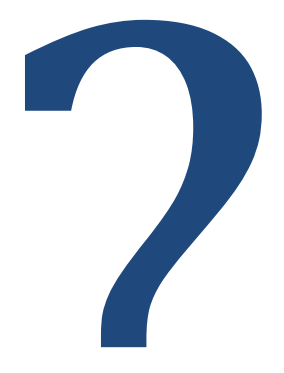

$27 / 27$ ! 